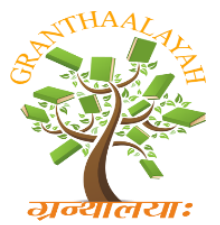

\author{
INTERNATIONAL JOURNAL OF RESEARCH - \\ GRANTHAALAYAH \\ A knowledge Repository
}

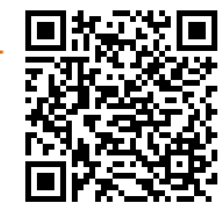

\title{
DETECTION OF HEPATITIS ANTIGEN AND ANTIBODY IN SERUM OF FEMALE HUMAN POPULATION
}

\author{
Rekha Khanna \\ Madhav Science P.G. Collage, Ujjain
}

\begin{abstract}
Immunization with hepatitis B (HB) vaccine is highly effective; however, more needs to be learned about the duration of protection and the need of booster dose. Present study suggest that age of vaccination is very important also it is observed that there is waning effect of vaccine after a long period. Our study throw light on the reliability of qualitative and quantitative tests to observe seropositivity of HBs antibodies. Qualitative detection of HBsAg was done with the help of chromatographic Hepacard method. For HBsAb detection we have done Immunochromatographic assay (Westernblotting). In order to monitor successful vaccination, chemiluminescent method is used to determine quantitatively HBsAb in the serum. The chromatographic methods are sensitive for the presence of antibody titer in serum below $100 \mathrm{mIU} / \mathrm{ml}$. Therefore Enzyme linked chemiluminiscent method is very sensitive for quantitative determination of HBsAb titre and thereby informative for the requirement of booster dose of vaccination.
\end{abstract}

Keywords:

Hepatitis Antigen, Antibody, Serum, Female.

\section{INTRODUCTION}

Hepatitis $\mathrm{b}$ virus is responsible for the most serious form of disease transmitted through sexual contacts, blood and other body fluids (1). Hepatitis B surface Ag is usually detected around 2-6 weeks in advance of clinical and biochemical evidence of hepatitis and persists throughout a clinical course of disease. Acute infection with hepatitis B virus is associated with acute viral hepatitis- an illness that begins with general ill-health, loss of appetite, nausea, vomiting, body aches, mild fever, dark urine, and then progresses to development of jaundice.

Several vaccines have been developed for the prevention of hepatitis B virus infection (2). These rely on the use of one of the viral envelope proteins (hepatitis B surface antigen or HBsAg). The vaccine was originally prepared from plasma (3) obtained from patients who had long-standing hepatitis B virus infection. However, currently, these are more often made using recombinant DNA technology (4), though plasma-derived vaccines continue to be used; the two types of vaccines are equally effective and safe. Vaccine is generally administered in either a two, three, or four dose schedules; and can be received by infants to adults.

Present study is focused on following parameters:

1. The effectiveness of vaccination after lapse of time period in different age of females.

2. Detection of total immunoglobulins (isotypes) involved in production of antibodies and their detection by reliable diagnostic method. 


\section{MATERIALS AND METHODS}

Serum samples of confirmed vaccinated volunteers of age groups 3 to 60 years were collected from different hospitals of Ujjain and categorized according to their age, and year of vaccination. All the samples were tested using different methods for the presence of $\mathrm{HBsAg}, \mathrm{HBcAB}$ and $\mathrm{HBsAb}$. Following methods are used to screen the serum samples for determining antibody titers in persons vaccinated with $\mathrm{HBs} \mathrm{Ag}$ :

\section{Methods of Detection of HBsAg and HBsAb in serum}

Serum samples were analyzed for qualitative detection of HBsAg with the help of Hepacard method as per the method mentioned in J. Mitra \& Co. Pvt. Ltd. It is a chromatographic visual, rapid and one step sandwich immunoassay for detecting HBsAg. In this method, monoclonal antibodies conjugated to colloid gold and polyclonal antibodies immobilized on a nitrocellulose strip. Serum sample flows through an absorbant pad and mixes with signal reagent. If the sample contains antigen, the colloid gold antibody conjugate and binds to the antigen, forming a complex. While migration of the complex through nitrocellulose strip, immobilized polyclonal antibody traps and form antibody- antigen-antibody colloidal gold coloured complex.

After screening the test serum samples for $\mathrm{HBsAg}$, following two methods are adopted for $\mathrm{HBsAb}$ detection:

$\mathbf{1}^{\text {st }}$ Method- Immunochromatographic assay For HBsAb (6) (Westernblotting) : It is two site sandwich assay that is used to detect the presence of HBsAb and is useful to monitor success of therapy from an acute HBV infection, determine past exposure to hepatitis b surface antigen and to test for immunity for hepatitis $b$. The kit contain Nitrocellulose membrane test assembly impregnated with colloidal gold conjugated to $\mathrm{Hbs} \mathrm{Ag}$ and mouse $\mathrm{IgG}$ antibodies, $\mathrm{HbsAg}$ and goat antimouse $\operatorname{IgG}$ at the respective regions. As the test specimen flows through nitrocellulose membrane the HBsAg colloidal gold conjugate complexes with HBsAb in the test specimen and travels on the membrane due to capillary action along with mouse $\mathrm{IgG}$ colloidal gold conjugate. This conjugate moves further on the membrane where it is immobilized by HBsAg (which is also coated on the membrane). A coloured band is formed at the test region (T). Absence of this band at the test region indicate the negative result. The mouse $\operatorname{IgG}$ colloidal gold conjugate and unbound complex if any move further on the membrane and are subsequently immobilized by the goat antimouse $\mathrm{IgG}$ antibodies coated on the membrane at the control region $\odot$ forming coloured band. $2^{\text {nd }}$ Method- Chemiluminescent method - the method is used to determine quantitatively HBsAb in human serum in order to monitor successful vaccination by using Architect anti-HBs assay (Abbott Laboratories). In this method HBsAg is coated with paramagnetic microparticals which combine with antiHBs present in the serum. After washing the acridium labeled HBsAg conjugate is added to the sample. Again after washing pretrigger and trigger solutions are added to the reaction mixture. Resulting chemiluminiscent reaction is measured as reactive light units (RLUs). A direct relationship exists between the amount of anti-HBs in the sample and the RLUs detected by the Architect system optics, Concentration of $\mathrm{HBsAb}$ is determined with calibration curve .If the concentration of specimen is greater than $10 \mathrm{mIU} / \mathrm{ml}$, the specimen is considered reactive for anti HBs.

\section{RESULTS AND DISCUSSION}

The comparison of methods for detection of HBsAg and HBsAb indicate that quantitative methods are more sensitive than chromatographic methods based on Western Blotting (qualitative). These 
chromatographic methods are sensitive for the presence of antigen or antibody titer in serum below $100 \mathrm{mIU} / \mathrm{ml}$. Chemiluminiscent method for the detection of HBsAb titre is very sensitive although the method require heavy setup of equipments and very sophisticated lab facilities.

In our studies the samples which were positive for $\mathrm{HBsAg}$ and $\mathrm{HBcAb}$ were not considered for $\mathrm{HBs} \mathrm{Ab}$ titre detection as they indicate natural HBsAg infection.

Our study revealed that out of 30 females vaccinated 10 years back, only $50 \%$ showed seropositive results as shown in Graph 1, 2 and 3. An antibody level between 10 and $100 \mathrm{mIU} / \mathrm{ml}$ is considered a poor response, which is detected by chromatographic method. Females of age group above 50 years showed very low HBsAb titers, as observed by quantitative method. Our studies support the early findings by Norouzirad, R. (8) that the level of anti-hepatitis B surface antigen antibodies (anti $\mathrm{HBs} \mathrm{Ab}$ ) declines over years after vaccination. Also, those volunteers vaccinated in govt. hospitals showed poor antibody titers. Out of total observed adolescent females $38 \%$ showed nonreactive values of $\mathrm{HBs} \mathrm{Ab}$ titers. Thus age of vaccination is very important. The amount of $\mathrm{Ab}$ synthesized due to Vaccination is analyzed by the use of graphs and other statistical methods.

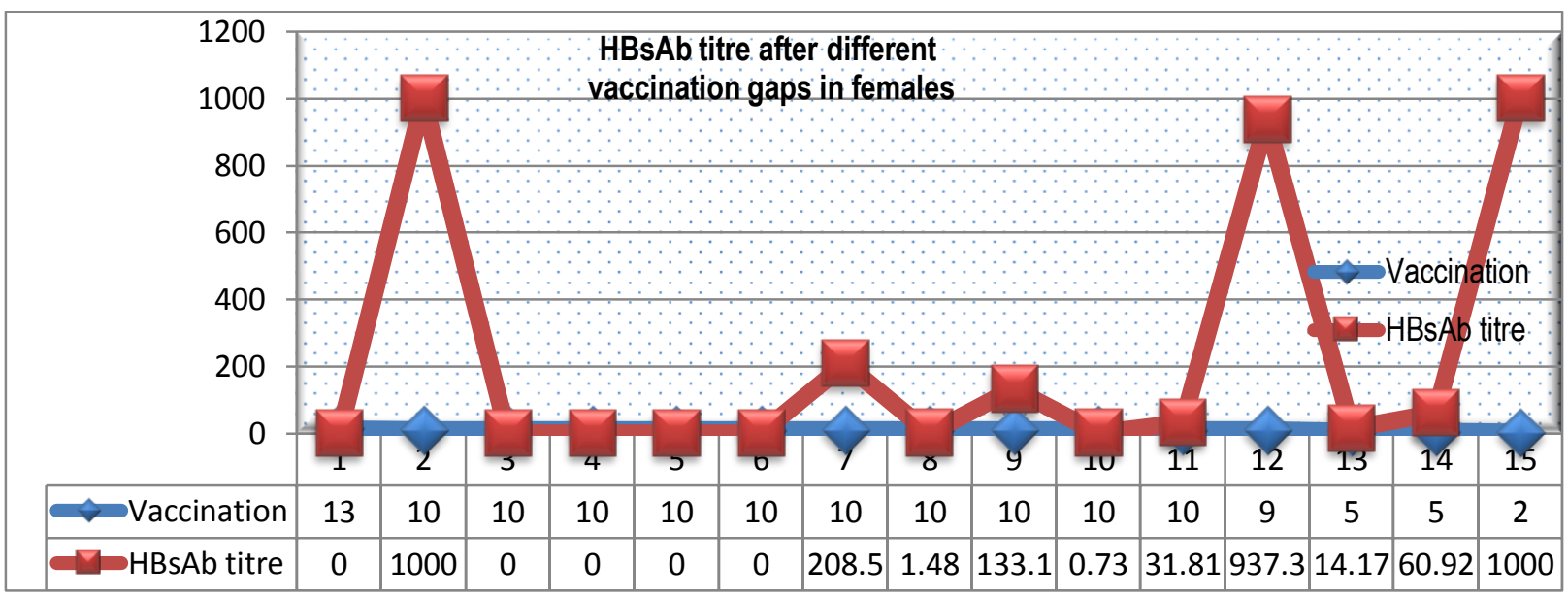

Graph 1: showing HBsAb titre after different vaccination gaps in females

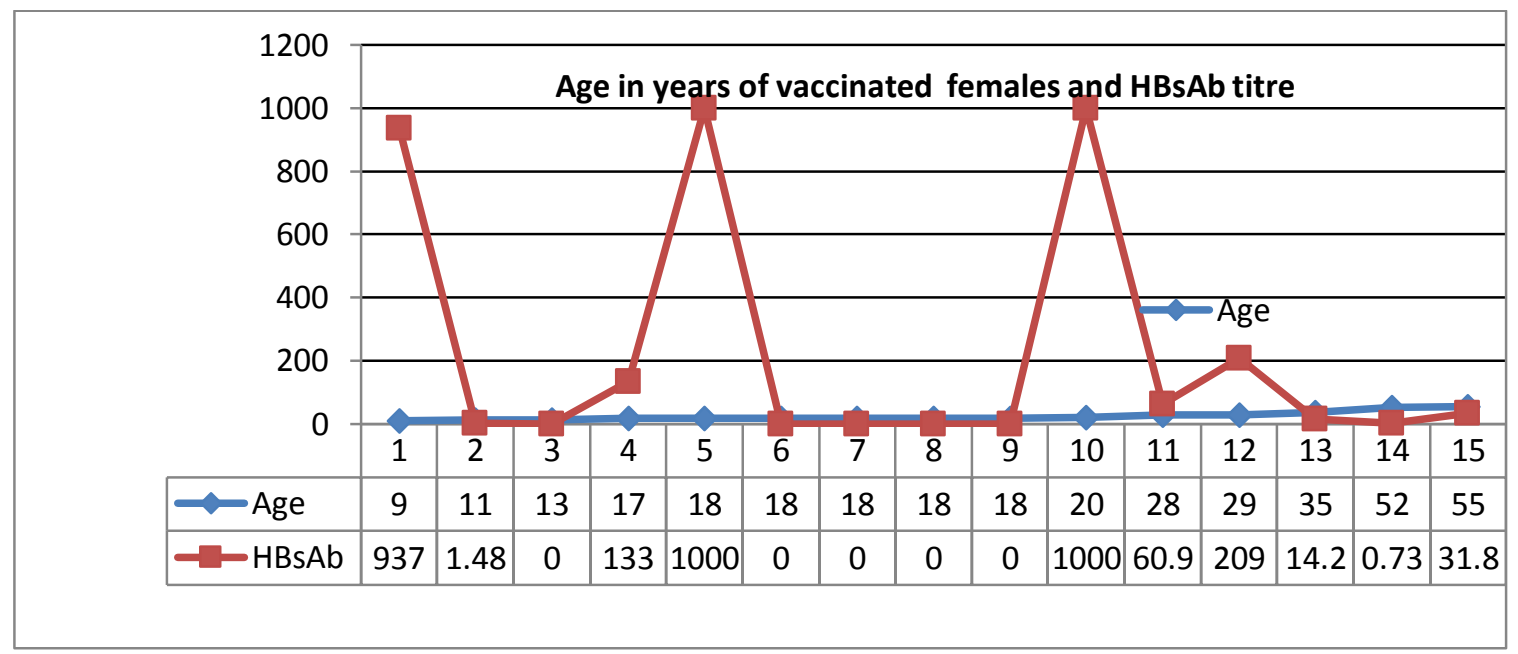

Graph 2: Showing HBsAb titre in different age groups of vaccinated females 


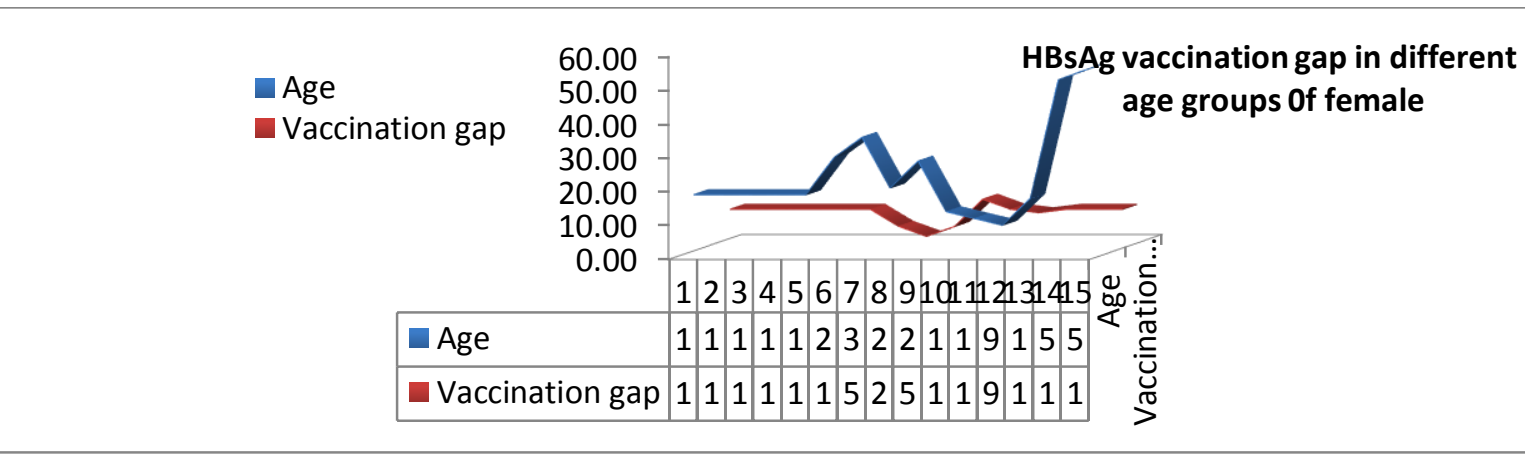

Graph 3: showing HBsAg vaccination gap in Females of different age groups

\section{Graph indicates that for the response of HBsAg vaccination, age of individual is responsible for HbsAb level.}

\section{REFERENCES}

1. Lavanchy, D., 2004. Hepatitis B virus epidemiology, desease burden, treatment and current imaging prevention and measures. J. Viral Hepat. 11, 97-107.

2. Janzen, L., Minuk, G.Y., Fast, M., Bernstein, K.N., 1996.Vaccine-induced hepatitis B surface antigen positivity in adult hemodialysis patients: Incidental and surveillance data. J. Am. Soc.Nephrol.7,1228-34.

3. Gerety, R. J., Ellis, R. W., 1987.Plasma-derived vs recombinant Hepatitis $B$ vaccine .Jama. 258(11).

4. Keating, G.M., Noble, S., 2003.Recombinant hepatitis B vaccine (Engerix-B): a review of its immunogenicity and protective efficacy against hepatitis B. Drugs. 63(10), 1021-51.

5. Mitra,J. \& Co. Pvt. Ltd. Hepacard: One step rapid visual test for the qualitative detection of HBsAg in human serum/plasma.

6. Hazarika, P., Online 2005. Sensitive detection of proteins using difunctional DNA-gold nanoparticles small, vol. 1, (8-9), , 844-848, XP002407187,

7. Anthony,G.D., Campbell,K., Hallett, M.B., Online: 2006. Chemiluminescence as an analytical tool in cell biology and medicine, DOI: 10.1002/9780470110522, ch7, John Wiley \& Sons, Inc.

8. Norouzirad,R., 2014. Serum levels of anti-Hepatitis B surface antibody among vaccinated population aged 1 to 18 years in Ahvaz city Southwest of Iran. Hepatitis 14(1):e13625. DOI: 10.5812/hepatmon.13625 PubMed 